\title{
Early anticoagulation for recurrent ischemic stroke despite the presence of ventricular bleeding
}

\begin{abstract}
In the setting of acute cardioembolic stroke, poor evidence exists on the use of anticoagulation early after the event, as well as in the presence of intraventricular bleeding. Moreover, the best timing to start therapy remains controversial for the high risk of hemorrhagic transformation (HT). Here, we describe the case of an 88-year-old man with atrial fibrillation (AF) and an acute ischemic stroke, who experienced early ischemic stroke recurrence and intraventricular bleeding after thrombolysis. Despite intraventricular bleeding and in light of the reduced kidney function, the decision was made to administer rivaroxaban $15 \mathrm{mg} /$ die starting 5 days after the index event. The early use of rivaroxaban $15 \mathrm{mg} /$ die was associated with clinical improvement in the absence of symptomatic HT and radiological worsening of intraventricular bleeding, and the pre-existing bleeding was regularly reabsorbed. Notably, 1 year after the ischemic stroke, the patient was still on oral anticoagulation with rivaroxaban $15 \mathrm{mg} / \mathrm{die}$, without occurrence of hemorrhagic complications. To our knowledge, this is the first evidence of the efficacy and safety of early anticoagulation with rivaroxaban $15 \mathrm{mg} / \mathrm{die}$ after an acute ischemic stroke and early stroke recurrence despite the presence of intraventricular bleeding.
\end{abstract}

Keywords: atrial fibrillation, direct oral anticoagulants, intraventricular bleeding, ischemic stroke, rivaroxaban
Volume 7 Issue 7 - 2017

\author{
Carolina Gentile,' Alessandro Adami \\ 'Neurology Clinic, S Maria della Misericordia University \\ Hospital, Italy \\ ${ }^{2}$ Stroke Center, Sacro Cuore Don Calabria Hospital, \\ Negrar, Italy
}

Correspondence: Carolina Gentile, Neurology Clinic, S. Maria della Misericordia University Hospital Piazzale Santa Maria della Misericordia, I 5 33I Udine, Italy, Tel +39 04322559020, Fax +39 04322559020, Email gentile.carolina2017@gmail.com

Received: October 12, 2017 | Published: November 27, 2017
Abbreviations: AF, atrial fibrillation; OAT, oral anticoagulant therapy; sHT, symptomatic hemorrhagic transformation; DOACs, direct oral anticoagulants; VKAs, vitamin $\mathrm{k}$ antagonists; NIHSS, national institute of health stroke scale; CT, computed tomography; MCA, middle cerebral artery; TIA, transient ischemic attack

\section{Introduction}

Ischemic stroke due to atrial fibrillation (AF) is associated with a high risk of recurrence, which may occur early after the index event. ${ }^{1}$ Oral anticoagulant therapy (OAT) is effective in preventing stroke recurrence and should be initiated as soon as possible. ${ }^{2}$ However, the choice of the best timing to start OAT remains controversial and requires an accurate evaluation of the patient clinical conditions, to limit the risk of symptomatic hemorrhagic transformation (sHT).

Direct Oral Anticoagulants (DOACs) are associated with a lower risk of hemorrhagic complications, especially intracranial bleeding, compared to vitamin $\mathrm{K}$ antagonists (VKAs). ${ }^{3}$ However, randomized clinical trials on DOACs have systematically excluded patients with recent ischemic stroke ( $<7$ days) in whom the risk of sHT is higher. ${ }^{4-7}$ Despite the lack of conclusive clinical evidence, the available data from observational, longitudinal studies suggest that the early use of DOACs is safe and might reduce the risk of $\mathrm{sHT}^{8-13}$ Therefore, in clinical practice, these agents are perceived as safe and increasingly used even during the first days after ischemic stroke.

Here, we report the case of a patient with an acute cardioembolic stroke, who received prompt anticoagulation with rivaroxaban $15 \mathrm{mg} /$ die for early ischemic stroke recurrence despite the presence of intraventricular bleeding.

\section{Case presentation}

An 88-year-old man with a history of hypertension and dyslipidemia, on treatment with an angiotensin-converting-enzyme inhibitor (5 $\mathrm{mg}$ ramipril), statin (40 $\mathrm{mg}$ atorvastatin), and antiplatelet therapy (100 mg acetylsalicylic acid), was admitted to our hospital with acute onset aphasia and weakness on the right side of the body (National Institute of Health Stroke Scale, NIHSS =13). At admission, a brain computed tomography $(\mathrm{CT})$ scan was performed, that showed signs of vascular attenuation in the M1 segment of the left middle cerebral artery (MCA), without signs of hemorrhagic and ischemic lesions (Figure 1A). The patient received intravenous thrombolysis within 3 hours from symptom onset. He was then transferred to the Stroke Unit, and a net improvement of the neurological deficit was observed in few days. Prolonged cardiac monitoring showed episodes of paroxysmal AF previously unknown.

The brain CT scan performed 24 hours after thrombolysis was still negative for parenchymal ischemia, while showing asymptomatic bilateral bleeding in the occipital horns of the lateral ventricles (Figure 1B). On the following day, the brain CT scan confirmed the presence of intraventricular bleeding, with signs of hypodense tissue in the terminal area of the left MCA inferior branch and of vascular hyperattenuation of the cortical branches of the left MCA (Figure 1C \& 1D). On day 5, aphasia suddenly worsened. A brain CT scan unveiled the presence of a new ischemic lesion in the left MCA inferior branches (Figure 1E \& 1F).

Due to the early ischemic recurrence, the decision was made to start anticoagulant therapy despite the presence of ventricular bleeding, and in the absence of contraindications to the use of rivaroxaban, ${ }^{14}$ it was deemed, at the dose of $15 \mathrm{mg} / \mathrm{die}$, as the optimal strategy due to the patient's reduced glomerular filtration rate $(34 \mathrm{ml} / \mathrm{min}){ }^{5,15}$ During the following days, the neurological conditions of the patient improved, with amelioration of both motor and language symptoms. On day 11, a brain CT demonstrated the reabsorption of the intraventricular bleeding without occurrence of new ischemic lesions (Figure 1G). On discharge, the NIHSS score was 2 and the patient was prescribed home treatment with rivaroxaban. At the 3-month follow-up, the patient had resumed his occupation as farmer and, 1 year after the ischemic stroke, he was still on oral anticoagulation with rivaroxaban $15 \mathrm{mg} /$ die, without reporting hemorrhagic complications. 

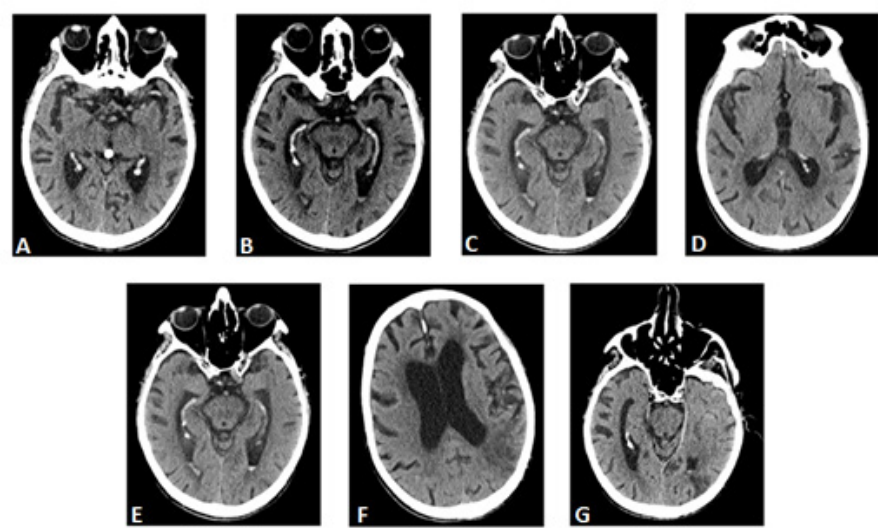

Figure I Brain CT scans. (A) Before thrombolysis; (B) 24h after thrombolysis, showing intraventricular bleeding; (C) 48h after thrombolysis, showing intraventricular bleeding and (D) Signs of distal MCA hyperattenuation; (E) 5 days after thrombolysis, showing intraventricular bleeding; (F) A new ischemic lesion; (G) II days after thrombolysis, showing reduction of intraventricular bleeding.

\section{Discussion}

In patients with acute cardioembolic stroke, the best time to start anticoagulation remains controversial due to the risk of sHT. As DOACs display a lower intracranial bleeding risk compared to the VKAs, initiation of DOAC therapy has been proposed to follow the 'rule of thumb' of 1-3-6-12: 1 day after a transient ischemic attack (TIA), 3 days after a non-disabling stroke, 6 days after a moderate stroke, and at least 12 days after a severe stroke. ${ }^{14}$ However, additional clinical evidence is needed to draw definitive conclusions on this crucial issue.

Here, we describe the case of an elderly AF patient with an acute ischemic stroke, who required prompt anticoagulation for early recurrence. As advanced age is not a contraindication to the use of rivaroxaban,,$^{14}$ he was treated with this DOAC at the dose of 15 $\mathrm{mg} /$ die starting 5 days after the index event, despite the presence of intraventricular bleeding occurred after the thrombolytic therapy for the first event. Our results are even more encouraging given that randomized clinical trials with DOACs have excluded acute ischemic stroke patients, and the safety of DOAC administration soon after an index event has only been addressed by observational, prospective, and non-randomized studies. ${ }^{8-13}$ In particular, in two prospective studies, the treatment with DOACs was commenced after a mean time of four and five days from the index event and no intracranial bleeding was observed during hospitalization, ${ }^{8,13}$ suggesting the safety of the early use of DOACs after an ischemic episode. Furthermore, the early use of DOACs seems to have the best safety profile when compared with other anticoagulants. ${ }^{11}$ Based on these data and in consideration of the patient's reduced kidney function, we opted for rivaroxaban $15 \mathrm{mg} / \mathrm{die}^{5,15}$ despite the presence of intraventricular bleeding and the lack of data on the effects of DOACs in case of such a complication. Moreover, little evidence describes the use of anticoagulation in the presence of HT of ischemic lesions. ${ }^{12}$ In a recent prospective study including AF patients treated with rivaroxaban $(20 \mathrm{mg}$ in $64 \%$ of cases) $\leq 14$ days from TIA or ischemic stroke (NIHSS $<9$ ), despite the small sample size $(n=60)$, the magnetic resonance imaging performed at baseline and 7 days after the start of therapy showed that no patient developed sHT of ischemic lesions. ${ }^{12}$ The case described here presented no HT of ischemic lesions but intraventricular bleeding; still, we observed that the early use of rivaroxaban $15 \mathrm{mg} / \mathrm{die}$, started 5 days after the recurrence of ischemic stroke, did not increase the risk of intracranial bleeding or of lesion HT. Rather, the use of rivaroxaban was associated with clinical improvement in the absence of radiological worsening of the ventricular bleeding and sHT, and the pre-existing bleeding was regularly reabsorbed. Our observation supports the hypothesis that the early use of rivaroxaban does not significantly increase the risk of intracranial bleeding or modify the course of the lesion.

Further studies are needed to validate the safety of the early use of rivaroxaban $15 \mathrm{mg} / \mathrm{die}$ and to determine the appropriate timing for therapy start after an acute ischemic stroke.

\section{Acknowledgements}

Financial support for editorial services was provided by Bayer HealthCare, Italy. Bayer HealthCare was not involved in the collection, analysis, and interpretation of data. The authors would like to thank Clara Ricci, PhD (Primula Multimedia SRL, Pisa, Italy) who provided skillful editorial assistance.

\section{Conflict of interest}

The authors have no financial disclosures or conflict of interest to report.

\section{Patient consent form}

The patient provided written informed consent and gave the permission for this case report.

\section{Funding}

None.

\section{References}

1. Stroke Risk in Atrial Fibrillation Working Group. Independent predictors of stroke in patients with atrial fibrillation: a systematic review. Neurology. 2007;69(6):546-554.

2. Kernan WN, Ovbiagele B, Black HR, et al. American Heart Association Stroke Council, Council on Cardiovascular and Stroke Nursing, Council on Clinical Cardiology, and Council on Peripheral Vascular Disease, Guidelines for the prevention of stroke in patients with stroke and transient ischemic attack: a guideline for healthcare professionals from the American Heart Association/American Stroke Association. Stroke. 2014;45(7):2160-2236.

3. Ruff CT, Giugliano RP, Braunwald E, et al. Comparison of the efficacy and safety of new oral anticoagulants with warfarin in patients with atrial fibrillation: a meta-analysis of randomised trials. Lancet. 2014;383(9921):955-962.

4. Connolly SJ, Ezekowitz MD, Yusuf S, et al. RE-LY Steering Committee and Investigators, Dabigatran versus warfarin in patients with atrial fibrillation. N Engl J Med. 2009;361(12):1139-1151.

5. Patel MR, Mahaffey KW, Garg J, et al. ROCKET AF Investigators, Rivaroxaban versus warfarin in nonvalvular atrial fibrillation. $N$ Engl $J$ Med. 2011;365(10):883-891.

6. Granger $\mathrm{CB}$, Alexander JH, McMurray JJ, et al. ARISTOTLE Committees and Investigators, Apixaban versus warfarin in patients with atrial fibrillation. $N$ Engl J Med. 2011;365(11):981-992.

7. Giugliano RP, Ruff CT, Braunwald E, et al. ENGAGE AF-TIMI 48 Investigators, Edoxaban versus warfarin in patients with atrial fibrillation. N Engl J Med. 2013;369(22):2093-2104.

8. Seiffge DJ, Traenka C, Polymeris A, et al. Early start of DOAC after ischemic stroke: Risk of intracranial hemorrhage and recurrent events. Neurology . 2016;87(18):1856-1862. 
9. Cappellari M, Carletti M, Danese A, et al. Early introduction of direct oral anticoagulants in cardioembolic stroke patients with non-valvular atrial fibrillation. $J$ Thromb Thrombolysis. 2016; 42(3):393-398.

10. Macha $\mathrm{K}$, Volbers $\mathrm{B}$, Bobinger $\mathrm{T}$, et al. Early Initiation of Anticoagulation with Direct Oral Anticoagulants in Patients after Transient Ischemic Attack or Ischemic Stroke. J Stroke Cerebrovasc Dis. 2016;25(9):2317-2321.

11. Paciaroni M, Agnelli G, Falocci N, et al. Early Recurrence and Cerebral Bleeding in Patients With Acute Ischemic Stroke and Atrial Fibrillation: Effect of Anticoagulation and Its Timing: The RAF Study. Stroke. 2015;46(8):2175-2182.

12. Gioia LC, Kate M, Sivakumar L, et al. Early Rivaroxaban Use After Cardioembolic Stroke May Not Result in Hemorrhagic Transformation: A Prospective Magnetic Resonance Imaging Study. Stroke. 2016;47(7):1917-1919.
13. Toyoda K, Arihiro S, Todo K, et al. SAMURAI Study Investigators, Trends in oral anticoagulant choice for acute stroke patients with nonvalvular atrial fibrillation in Japan: the SAMURAI-NVAF study. Int J Stroke. 2015;10(6):836-842.

14. Heidbuchel H, Verhamme P, Alings M, et al. Updated European Heart Rhythm Association Practical Guide on the use of non-vitamin K antagonist anticoagulants in patients with non-valvular atrial fibrillation. Europace. 2015;17(10):1467-1507.

15. http://www.ema.europa.eu/docs/en_GB/document_library/EPAR Product_Information/human/000944/WC500057108.p̄df. 